\title{
Using Hidden Markov Models for Recognizing Action Primitives in Complex Actions
}

\author{
Volker Krüger and Daniel Grest \\ Computer Vision and Machine Intelligence Lab \\ CIT, Aalborg University \\ Lautrupvang 15 \\ 2750 Ballerup \\ Denmark \\ vok@cvmi . aau . dk
}

\begin{abstract}
There is biological evidence that human actions are composed out of action primitives, like words and sentences being composed out of phonemes. Similarly to language processing, one possibility to model and recognize complex actions is to use grammars with action primitives as the alphabet. A major challenge here is that the action primitives need to be recovered first from the noisy input signal before further processing with the action grammar can be done. In this paper we combine a Hidden Markov Model-based approach with a simplified version of a condensation algorithm which allows to recover the action primitives in an observed action. In our approach, the primitives may have different lengths, no clear "divider" between the primitives is necessary. The primitive detection is done online, no storing of past data is required. We verify our approach on a large database. Recognition rates are slightly lower than the rate when recognizing the singular action primitives.
\end{abstract}

\section{Introduction}

There is biological evidence that actions and activities are composed out of action primitives similarly to phonemes being concatenated into words 21/20].

In this sense, one can define a hierarchy of action primitives at the coarsest level, and then actions and activities as the higher abstract levels where actions are composed out of the action primitives while activities are, in turn, a composition of the set of actions 2171. If the action primivies are used as an alphabet one can use action grammars 12,23 to model actions and activities. It is an open problem how to define and detect these action primitives and how to define these grammars. It is reasonable to assume that these things can only be defined in context of the specific application at hand.

If an observed complex action is given and a grammar should be used for parsing and recognition, then the first necessary step is to recover the letters in

${ }^{1}$ In the following, we define the term action as a sequence of action primitive of arbitrary length. 
this observed action, i.e., the action primitives. Once the observed (continuous) sequence has been translated into a discrete set of symbols (letters), parsing based on the grammar description can be done.

In other words, if we have given an alphabet of action primitives $P$ and if we define any action $O$ to be a composition $O=a_{1} a_{2} a_{3} \ldots a_{T}$ of these action primitives, then our goal is to recover these primitives and their precise order. The same problem is also found in speech recognition where the goal is to find the right sequences of phonemes (see Sec. 2). Once we have recovered the sequence of action primitives in the observed sequence, we can identify the action through parsing. (In speech recognition, the sequence of detected phonemes is used to identity the corresponding word.)

The recovery of the action primitives is a non-trivial problem. Unlike phonemes (see also discussion in Sec. 2), action primitives can have a "long" durations and the variance of their execution speed may vary greatly. Also, action primitives can be heavily smeared out which complicates the distinction between them.

In this paper we deal with the recovery of the sequence of the action primitives out of an action, when a set (or alphabet) of action primitives is given.

In order to take into account possible noise and imperfect data, we base our approach on Hidden Markov Models (HMMs) 918 and represent our action primitives with HMMs.

Thus, given a set of action primitives $P$ where each action primitive is represented by an HMM and given an observed sequence $O$ of these action primitives where

1. the order of the action primitives and

2. the duration of each single action primitive and the position of their boundaries

are unknown, we would like to identify the most likely sequence of action primitives in the observation sequence $O$.

According to the biological findings, the representation for action recognition is closely related to the representation for action synthesis (i.e. the motor representation of the action) 21720]. This motivates us to focus our considerations in this paper to actions represented in joint space. Thus, our actions are given as sequences of joint settings. A further justification for this approach is that this action representation can then be used, in future work, to bias 3D body trackers as it operates directly on the $3 \mathrm{D}$ parameters that are to be estimated by the $3 \mathrm{D}$ tracker. However, our focus on joint data is clearly without limiting generality and our technique can be applied also to other types of action representations as long as the features live in a metric space. In our on-going research we have applied the same techniques of this paper also to action recognition based on silhouettes 15 .

This paper is structured as follows: In Sec. 2 will will give an overview of related work. In Sec. 3 we will discuss our approach for the HMM-based recognition of the action primitives. In Sec. 4 we present our extensive experimental results. The paper is concluded then in Sec. 5 with final comments. 


\section{Related Work}

The recovery of phonemes in speech recognition is a closely related to our problem and thus the techniques applied there wore worthwhile to be investigated. In speech recognition, acoustic data gets sampled and quantized, followed by using Linear Predictive Coding (LPC) to compute a cepstral feature set. Alternatively to LPC, a Perceptual Linear Predictive (PLP) analysis 8 is often applied. In a later step, time slices are analyzed. Gaussians are often used here to compute the likelihoods of the observations of being a particular phoneme 10. An alternative way to the Gaussians is to analyze time slices with an Artificial Neural Network 3 . Timeslices seem to work well on phonemes as the phonemes have usually a very short duration. In our case, however, the action primitives have usually a much longer duration and one would have to deal with a combinatorial problem when considering longer time slices.

In the following we will shortly review the most recent publications that consider the action recognition problem based on action primitives.

As mentioned above, the human visual system seems to relate the visual input to a sequence of motor primitives when viewing other agents performing an action 21 7/20]. These findings have gained considerable attention from the robotics community 226]. In imitation learning the goal is to develop a robot system that is able to relate perceived actions to its own motor control in order to learn and to later recognize and perform the demonstrated actions.

In 1413, Jenkins et al. suggest applying a spatio-temporal non-linear dimension reduction technique on manually segmented human motion capture data. Similar segments are clustered into primitive units which are generalized into parameterized primitives by interpolating between them. In the same manner, they define action units ("behavior units") which can be generalized into actions. In [11 the problem of defining motor primitives is approached from the motor side. They define a set of nonlinear differential equations that form a control policy (CP) and quantify how well different trajectories can be fitted with these CPs. The parameters of a CP for a primitive movement are learned in a training phase. These parameters are also used to compute similarities between movements. In [514] a HMM based approach is used to learn characteristic features of repetitively demonstrated movements. They suggest to use the HMM to synthesize joint trajectories of a robot. For each joint, one HMM is used. In [5] an additional HMM is used to model end-effector movement. In these approaches, the HMM structure is heavily constrained to assure convergence to a model that can be used for synthesizing joint trajectories.

In [16, Lu et al. also approach the problem from a system theoretic point of view. Their goal is to segment and represent repetitive movements. For this, they model the joint data over time with a second order auto-regressive (AR) model and the segmentation problem is approached by detection significant changes of the dynamical parameters. Then, for each motion segment and for each joint, they model the motion with a damped harmonic model. In order to compare actions, a metric based on the dynamic model parameters is defined. An approach of using 
meaningful instants in time is proposed by Reng et al. [19] where key poses are found based on the curvature and covariance of the normalized trajectories.

\section{Representing and Recognizing Action Primitives Using HMMs}

In order to approach the action recognition problem, we model each of the action primitives $P=\left\{a^{1}, a^{2}, \ldots, a^{N}\right\}$ with a continuous mixture-HMM. A Hidden Markov Model (HMM) probabilistic version of a finite state machine. It is generally defined as a triplet $\lambda=(A, B, \pi)$, where $A$ gives the transition likelihoods between states, $B$ the observation likelihoods, conditioned on the present state of the HMM, and the starting state $\pi$ (see the classics [918] for a further introduction). In case of the continuous mixture HMM, the observation likelihoods are given as Gaussian mixtures with $M \geq 1$ mixtures.

Our HMMs are trained on demonstrations of different individuals and the Gaussian mixtures are able to capture the variability between them. The training results into a set of HMMs $\left\{\lambda_{i} \mid i=1 \ldots N\right\}$, one for each action primitive.

Once each action primitive is represented with an HMM, the primitives can generally simply be recognized with the classical recognition technique for HMMs, a maximum likelihood or a maximum a-posteriori classifier: Given an observation sequence $O_{t}$ of an action primitive, and a set of HMMs $\lambda_{i}$, the maximum likelihood (ML)

$$
\max _{i} P\left(O_{t} \mid \lambda_{i}\right)
$$

identifies the most likely primitive. An alternative to the ML technique is the maximum a-posteriori (MAP) estimate that allows to take into account the likelihood of observing each action primitive:

$$
\max _{i} P\left(\lambda_{i} \mid O_{t}\right)=\max _{i} P\left(O_{t} \mid \lambda_{i}\right) P\left(\lambda_{i}\right)
$$

where $P\left(\lambda_{i}\right)$ is the likelihood that the action, represented by the HMM $\lambda_{i}$ appears.

\section{Recognition with HMMs}

In general, the likelihood of an observation for some HMM $\lambda_{i}$ can be computed as

$$
\begin{aligned}
P\left(O \mid \lambda_{i}\right) & =\sum_{S} P\left(O, S \mid \lambda_{i}\right) \\
& =\sum_{S} P\left(O \mid S, \lambda_{i}\right) P\left(S \mid \lambda_{i}\right) \\
& =\sum_{S} \prod_{t=0}^{T} P\left(O_{t} \mid S_{t}, \lambda_{i}\right) \prod_{t=0}^{T} P\left(S_{t} \mid S_{t-1}, \lambda_{i}\right) .
\end{aligned}
$$


Here, one needs to marginalizes over all possible state sequences $S=\left\{S_{0}, \ldots, S_{T}\right\}$ the HMM $\lambda_{i}$ can pass through.

To apply this technique to our problem directly is difficult in our case: In Eq. 3.5 we evaluate at the end of the observation $O$ and select the HMM which explains this observation best. In our case, however, we are not able to identify when one primitive ends and where a new one starts. Thus, the problem is that we do not know when to evaluate, i.e. at what time steps $t$ we should stop and do the maximum-likelihood estimation to find the most likely action primitive that was just now being observed.

Instead of keeping the HMMs distinct, our suggestion is to insert the "action identifier" $i$ of the HMM $\lambda_{i}$ as a random variable into Eq. (5) and to rewrite it as

$$
P(O \mid a)=\sum_{S} \prod_{t=0}^{T} P\left(O_{t} \mid S_{t}, i_{t}\right) P\left(S_{t}, i_{t} \mid S_{t-1}, i_{t-1}\right) .
$$

In other words, we would like to estimate at each time step the likelihood of action $i$ and the state $S$ from the previously seen observations, or, respectively, the probability of $\lambda_{i}$ being a model of the observed action:

$$
P\left(S_{T}, i_{T} \mid O_{0: T}\right)=\prod_{t=0}^{T} P\left(O_{t} \mid S_{t}, i_{t}\right) P\left(S_{t}, i_{t} \mid S_{t-1}, i_{t-1}\right) .
$$

The difference in the interpretation becomes more clear when we write Eq. (77) in a recursive fashion:

$$
P\left(S_{t+1}, i_{t+1} \mid O_{0: t+1}\right)=P\left(O_{t+1} \mid S_{t+1}, i_{t+1}\right) P\left(S_{t+1}, i_{t+1} \mid S_{t}, i_{t}\right) P\left(S_{t}, i_{t} \mid O_{0: t}\right)
$$

This is the classical Bayesian propagation over time. It computes at each time step $t$ the likelihood of observing the action $i_{t}$ while having observed $O_{0: t}$. If we ignore the action identifier $i_{t}$, then Eq. (8) explains the usual efficient implementation of the forward algorithm [9]. Using the random variable $i_{t}$, Eq. (8) defines a pdf across the set of states (where the state vector $S_{t}$ is the concatenation of state vectors of each individual HMM) and the set of possible actions. The effect of introducing the action $i$ might not be obvious: using $i$, we do not any more estimate the likelihood of an observation, given a HMM $\lambda_{i}$. Instead, we compute at each time step the probability mass function (pmf) $P\left(S_{t}, i_{t} \mid O_{0: t}\right)$ of each state and each identity, given the observations. By marginalizing over the states, we can compute the pmf $P\left(i_{t} \mid O_{0: t}\right)$ for the action at each time step. The likelihood $P\left(i_{t} \mid O_{0: t}\right)$ converges to the most likely action primitive as time progresses and more data becomes available (see Fig. 1).From Fig. 1]it is apparent that the pmf $P\left(i_{t} \mid O_{0: t}\right)$ will remain constant after convergence as one action primitive will have the likelihood 1 and all other primitive likelihoods have vanished. To properly evaluate the entire observation sequence, we apply a voting scheme that counts the votes after each convergence and then restarts the HMMs. The states are initialized with the present observation likelihoods and then propagated with 


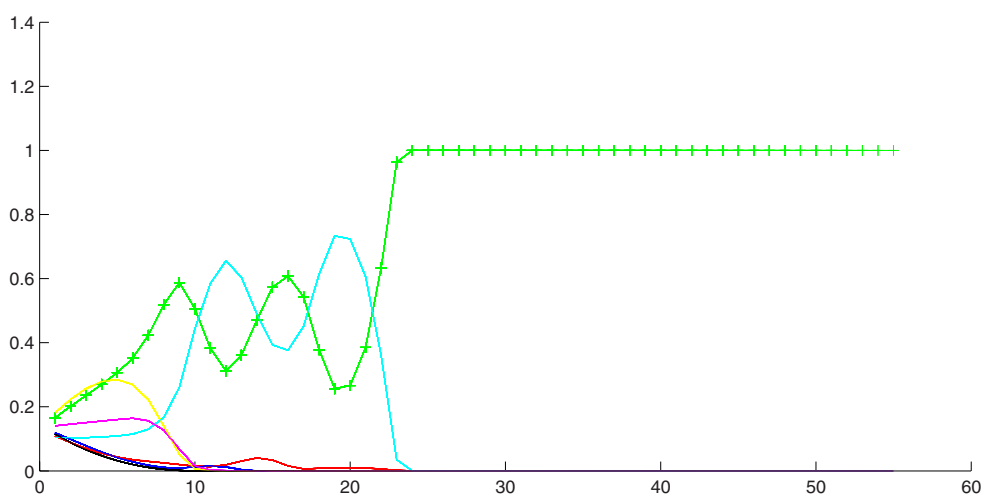

Fig. 1. shows an example for a typical behavior of the pmf $P\left(i_{t} \mid O_{0: t}\right)$ for each of the actions $i$ as time $t$ progresses. One can see that the likelihood for one particular action (the correct one in this example, marked with "+") converges to 1 while the likelihoods for the others vanish.

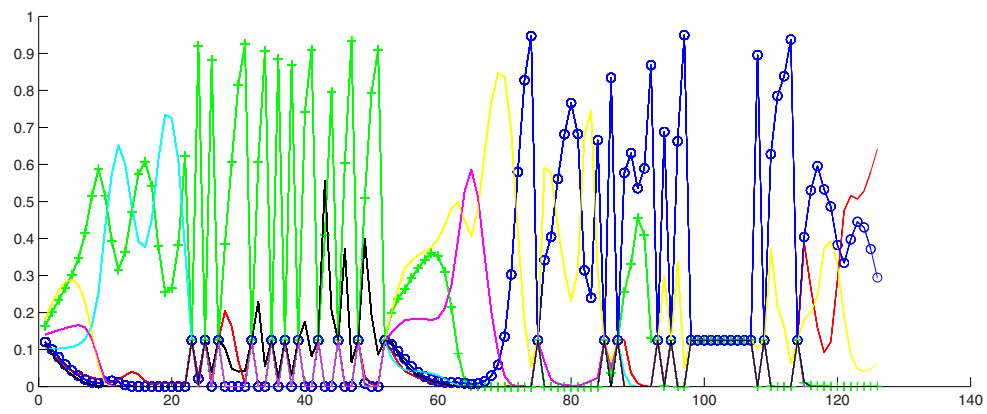

Fig. 2. shows an example for a typical behavior of the pmf $P\left(i_{t} \mid O_{0: t}\right)$ as time $t$ progresses. The input data consisted of two action primitives: first, action primitive " 2 ", marked with "+", then, action primitive "3", marked with "o". One can see that until $\approx$ sample 52 the system converges to action " 2 ", after sample 70 , the system converges to primitive 3 . The length of the first sequence is 51 samples, the length of sequence 2 is 71 samples.

the transition matrix as usual. Fig. 2 shows the repeated convergence and the restarting of the HMMs. In the example shown in Fig. 2 we have used two concatenated action primitives, denoted by the green curve with the "+" and by the blue curve with the "o", respectively. The first action primitive was in the interval between 0 and 51, while the second action primitive was from sample 52 to the end. One can see that the precise time step when primitive 1 ended and when primitive 2 started cannot be identified. But this does not pose a problem for our recovery of the primitives as for us the order matters but not their precise duration. In Fig. 1 a typical situation can be seen where the observed data did not give enough evidence for a fast recognition of the true action. 


\section{Experiments}

For our experiments, we have used our MoPrim [19] database of human one-arm movements. The data was captured using a FastTrack Motion capture device with 4 electromagnetic sensors. The sensors are attached to the torso, shoulder, elbow and hand (see Fig. 3). Each sensor delivers a $6 D$ vector, containing $3 D$ position and $3 D$ orientation thus giving a $24 D$ sample vector at each timestep ( 4 sensors with each $6 D$ ). The MoPrim database consists of 6 individuals, showing 9 different actions, with 20 repetitions each. The actions in the database are simple actions such as point forward, point up, "come here", "stop!". Each sequence consists of $\approx 60-70$ samples and each one starts with 5 samples of the arm in a resting position, i.e., it is simply hanging down.

Instead of using the sensor positions directly, we transform the raw $24 D$ sensor data into joint angles: one elbow angle, one shoulder angle between elbow, shoulder and torso and a 3D orientation of the normal of this shoulder-elbowtorso-triangle. The orientation of the normal is given with respect to the normal of this triangle when the arm is in resting position. All angles are given in radians. No further processing of the MoPrim data was done.

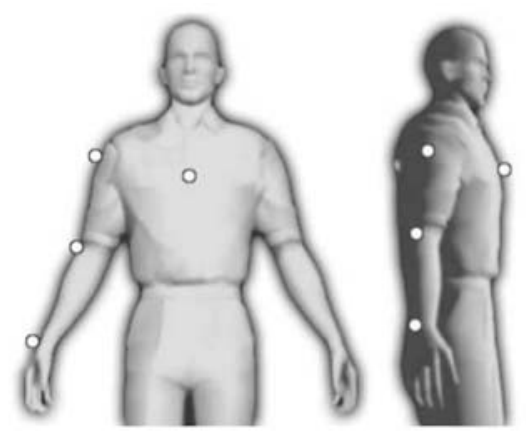

Fig. 3. marks the positions of the magnetic sensor on the human body

We have carried out several different experiments:

1. In the first test, we tested for invariance with respect to the performing human. We have trained nine HMM for nine action. Each of the HMMs was trained on 6 individuals and all the 20 repetitions of the actions. The recognition testing was then carried out on the remaining individual (leaveone-out-strategy). The HMMs we use were mixture HMMs with 10 states and 5 mixtures per state (these numbers are chosen arbitrarily, and they gave good results).

2. Here, we tested for invariance with respect to the variations within the repetitions. We have trained nine HMMs for nine actions. Each HMM was trained on all individuals but only on 19 repetitions. The test set consisted of the 20 th repetition of the actions. 
3. As a base line reference, we have tested how good the HMMs are able to recognize the actions primitives by testing action primitive sequences of length 1. Here, the HMMs were trained as explained under 2 above. This test reflects the recognition performance of the classical maximum-likelihood approach.

4. We have repeated the above three experiments after having added Gaussian noise with zero mean and a standard deviation of $\sigma=0, \sigma=0.3$ and $\sigma=1$ to the training and testing data. As all angles are given in radians, thus, this noise is considerable.

To achieve a good statistic we have for each test generated 10.000 test actions of random length $\leq 100$. Also, we have systematically left out each individual (action) once and trained on the remaining ones. The results below are averaged across all leave-one-out tests. In each test action, the action primitives were chosen randomly, identically and independently. Clearly, in reality there is a strong statistical dependency between action primitives so that our recognition results can be seen as a lower bound and results are likely to increase considerably when exploiting the temporal correlation by using an action grammar (e.g. another HMM).

The results are summarized in Table 1. One can see that the recognition rates of the individual action primitives is close to the general base-line of the HMMs. The recognition rates degrade with increasing noise which was to be expected, however, the degradation effect is the same for all three experiments (identities, repetition, baseline).

Table 1. summarizes the results of our various experiments. In the experiments, the training of the HMMs were done without the test data. We tested for invariance w.r.t. identity and w.r.t. the action. The baseline shows the recognition results when the test action was a single action primitives.

\begin{tabular}{|l|c|c|}
\hline \multicolumn{3}{|l|}{ Leave-one-Out experiments } \\
\hline Test & Noise $\sigma$ & Recognition Result \\
\hline Identities (Test 1) & 0 & 0.9177 \\
Repetitions (Test 2) & 0 & 0.9097 \\
Baseline (Test 3) & 0 & 0.9417 \\
\hline Identities (Test 1) & 0.5 & 0.8672 \\
Repetitions (Test 2) & 0.5 & 0.8710 \\
Baseline (Test 3) & 0.5 & 0.8649 \\
\hline Identities (Test 1) & 1 & 0.3572 \\
Repetitions (Test 2) & 1 & 0.3395 \\
Baseline (Test 3) & 1 & 0.3548 \\
\hline
\end{tabular}

All actions in the action database start and end in a resting pose. To assure that the resting pose does not effect the recognition results, we have repeated the above experiments on the action primitives where the rest poses were omitted. However, the recognition results did not change notably. 


\section{Conclusions}

In this work we have presented an approach to recover the motion primitives from an action where the motion primitives are represented with a Hidden Markov Model. The approach we have taken is to consider the joint distribution of the state and the action at the same time instead of using the classical maximum likelihood approach. The experiments show that the approach is able to successfully recover the action primitives in the action with a large likelihood. It is worth pointing out that in our experiments the pairwise appearance of action primitives was statistically independent. Thus, for the recovery of the action primitives no temporal constraints between the action primitives were used or exploited. Temporal constraints between the action primitives are later introduced at a higher level though action grammars.

In future work we will use a further HMM to learn sequences of action primitives from training examples to learn such an action grammar.

Acknowledgment. This work was partially funded by PACO-PLUS (IST-FP6IP-027657).

\section{References}

1. Billard, A., Epars, Y., Calinon, S., Schaal, S., Cheng, G.: Discovering Optimal Imitation Strategies. Robotics and Autonomous Systems 47, 69-77 (2004)

2. Bobick, A.F.: Movements, Activity, and Action: The Role of Knowledge in the Perception of Motion. In: Royal Society Workshop on Knowledge-based Vision in Man and Machine, London, England, February 1997 (1997)

3. Bourlard, H., Morgan, N.: Connectionist Speech Recognition: a Hybrid Approach. Kluwer Academic Publishers, Dordrecht (1994)

4. Calinon, S., Billard, A.: Stochastic Gesture Production and Recognition Model for a Humanoid Robot. In: International Conference on Intelligent Robots and Systems, Alberta, Canada, August 2-6, 2005 (2005)

5. Calinon, S., Guenter, F., Billard, A.: Goal-Directed Imitation in a Humanoid Robot. In: International Conference on Robotics and Automation, Barcelona, Spain, April 18-22, 2005 (2005)

6. Dariush, B.: Human Motion Analysis for Biomechanics and Biomedicine. Machine Vision and Applications 14, 202-205 (2003)

7. Giese, M., Poggio, T.: Neural Mechanisms for the Recognition of Biological Movements. Nature Reviews 4, 179-192 (2003)

8. Hermansky, H.: Perceptual linear predictive (plp) analysis of speech. Journal of Acoustical Society of America 87(4), 1725-1738 (1990)

9. Huang, X.D., Ariki, Y., Jack, M.A.: Hidden Markov Models for Speech Recognition. Edinburgh University Press (1990)

10. Huang, X.D., Jack, M.A.: Semi-continous hidden markov models for speech signals. Computer Speech and Language 3, 239-252 (1989)

11. Ijspeert, A.J., Nakanishi, J., Schaal, S.: Movement Imitation withNonlinear Dynamical Systems in Humanoid Robots. In: International Conference on Robotics and Automation, Washington DC, USA (2002) 
12. Ivanov, Y., Bobick, A.: Recognition of Visual Activities and Interactions by Stochastic Parsing. IEEE Transactions on Pattern Analysis and Machine Intelligence $22(8), 852-872(2000)$

13. Jenkins, O.C., Mataric, M.: Deriving Action and Behavior Primitives from Human Motion Capture Data. In: International Conference on Robotics and Automation, Washington DC, USA (2002)

14. Jenkins, O.C., Mataric, M.J.: Deriving Action and Behavior Primitives from Human Motion Data. In: International Conference on Intelligent Robots and Systems, pp. 2551-2556, Lausanne, Switzerland, September 30 - October 4, 2002 (2002)

15. Krueger, V., Anderson, J., Prehn, T.: Probabilistic model-based background subtraction. In: Scandinavian Conference on Image Analysis, pp. 180-187, June 19-22, Joensuu, Finland (2005)

16. Lu, C., Ferrier, N.: Repetitive Motion Analysis: Segmentation and Event Classification. IEEE Transactions on Pattern Analysis and Machine Intelligence 26(2), 258-263 (2004)

17. Nagel, H.-H: From Image Sequences Towards Conceptual Descriptions. Image and Vision Computing 6(2), 59-74 (1988)

18. Rabiner, L.R., Juang, B.H.: An introduction to hidden Markov models. IEEE ASSP Magazine, pp. 4-15 (1986)

19. Reng, L., Moeslund, T.B., Granum, E.: Finding Motion Primitives in Human Body Gestures. In: Gibet, S., Courty, N., Kamp, J.-F. (eds.) GW 2005. LNCS (LNAI), vol. 3881, pp. 133-144. Springer, Heidelberg (2006)

20. Rizzolatti, G., Fogassi, L., Gallese, V.: Parietal Cortex: from Sight to Action. Current Opinion in Neurobiology 7, 562-567 (1997)

21. Rizzolatti, G., Fogassi, L., Gallese, V.: Neurophysiological Mechanisms Underlying the Understanding and Imitation of Action. Nature Reviews 2, 661-670 (2001)

22. Schaal, S.: Is Imitation Learning the Route to Humanoid Robots? Trends in Cognitive Sciences 3(6), 233-242 (1999)

23. Stolcke, A.: An Efficient Probabilistic Context-Free Parsing Algorithm That Computes Prefix Probabilities. Computational Linguistics 21(2), 165-201 (1995) 